\title{
Investigation of interphase effects on mechanical behaviors of carbon nanocone-based composites
}

\author{
Mir Masoud Seyyed Fakhrabadi ${ }^{1, a}$ AND NAVID Khani ${ }^{2,3}$ \\ 1 Department of Mechanical Engineering, College of Engineering, Karaj Branch, Islamic Azad University, Karaj, Iran \\ 2 Faculty of Engineering and Natural Sciences, Sabanci University, Istanbul, Turkey \\ 3 School of Mechanical Engineering, College of Engineering, University of Tehran, Tehran, Iran
}

Received 16 February 2014, Accepted 16 May 2014

\begin{abstract}
This paper presents the mechanical properties of carbon nanocone-based polymer composites in the presence of interphase layer. Some representative volume elements are considered to study the elastic behaviors of the transversely isotropic nanocomposite and the effects of the interphase layer on four of five independent constants related to this model. The finite element method is applied to obtain the results for different elastic moduli and thicknesses of the matrix.
\end{abstract}

Key words: Carbon nanocone / polymer composite / interphase layer

\section{Introduction}

Development of composite materials during the past decades can be accounted a great progression in engineering. Mechanical and material engineers have attempted to enhance the quality and applicability of these materials from all possible facets. These activities lead to application of the composite materials in different engineering fields such as aviation technology, chemical and petrochemical complexes, refineries, car industries, etc. With increasing the demands of mentioned industries, researchers try to improve the mechanical behaviors of the composite materials with introducing and applying novel theoretical and experimental techniques such as application of new materials as matrix or filler of the composites. One of the main influential materials for this purpose is nanomaterials. Nano $\mathrm{Al}_{2} \mathrm{O}_{3}$, nano $\mathrm{Si}_{3} \mathrm{~N}_{4}$, nano clay and nano $\mathrm{SiO}_{2}$ are some exemplary nanoscale samples for fillers which were used and investigated by some researchers [1-3]. Although these nanomaterials enhance different aspects of the mechanical behaviors of the nanocomposites, one of the most underlying types of these nanomaterials is carbon nanostructures such as carbon nanotubes (CNTs), carbon nanocones (CNCs), buckyballs and carbon nanobuds (CNBs). They can be applied as fillers embedded in matrices such as polymers (resin, rubber, etc.) or metals (aluminum, titanium, etc.) to make up their deficiencies and enhance their operational properties. It is worth noting that these nanomaterials

${ }^{a}$ Corresponding author: msfakhrabadi@gmail.com do not affect the advantages of the matrices effectively. For instance, low density, high elastic modulus and high tensile strength of CNTs made them be important candidates to be used in nanocomposites [4]. This fact can be generalized to other carbon nanostructures due to their similar properties. Because studying the effects of CNC as one of the carbon nanostructures on the mechanical properties of nanocomposites is the goal of this paper, we only review the carbon-based composites.

Liu and Chen estimated the effective material properties of CNT-based composites through a nanoscale representative volume element (RVE) [5]. They supposed a transversely isotropic cylindrical RVE and applied finite element method (FEM) to evaluate four of five independent constants related to this model using several loading cases which are to be discussed in next sections. Another research was about application of square RVEs to study the effective material properties of CNT-based composites [6]. In this article, the effects of short and long CNTs on the mechanical properties of nanocomposites are presented in detail.

The length of CNTs possesses underlying effects on the efficiency of the nanocomposites. Shokrieh and Rafiee investigated this fact using a multiscale modeling method [7]. In their paper, a continuum model for matrix, a discrete model for CNT and linear elements instead of van der Waals interactions between the matrix and filler were applied. In an other study, Mokashi et al. investigated tensile response and fracture in CNT-based polyethylene composites via molecular mechanics (MM) approach [8]. The systems under investigation consisted 
of amorphous and crystalline polyethylene composites with embedded single-walled CNTs. They calculated the elastic moduli of amorphous and crystalline polyethylene through the mentioned method and verified them by experimental data.

Beside the elastic properties of CNT-based composites, other behaviors such as vibrations and thermal properties of these materials were studied. Formica et al. investigated the vibrations of three kinds of CNT-reinforced composites including epoxy, rubber, and concrete [9]. They applied an equivalent continuum model based on Eshelby-Mori-Tanaka method and evaluated the effective constitutive law of the elastic isotropic matrix with dispersed elastic CNTs. Furthermore, the effects of the CNT alignment and volume fraction were investigated to assess how the modal properties were influenced by these factors. In an other paper, Ke et al. studied the nonlinear free vibration of functionally graded CNT-reinforced composite beams based on Timoshenko beam model and von Karman geometric nonlinearity [10]. The material properties were considered to be graded in the thickness direction and estimated through rule of mixture. The Ritz method was applied to obtain the governing equation which was then solved by a direct iterative approach to derive the nonlinear vibration frequencies of the CNT-reinforced functionally graded micro beam.

As mentioned before, some researchers studied the effects of CNTs on the thermal properties of nanocomposites with different matrices $[11,12]$. But due to their less relation to the target of this paper, we do not review them in detail.

The reviewed papers were some exemplary samples of several papers related to CNT-based nanocomposites. These papers show that the mechanical behaviors of the CNT-based nanocomposites were studied well. But, these properties for other carbon nanostructures-based composites such as CNCs, carbon nanobuds and buckyballs were not researched in detail. C60 is a spherical molecule composed of sixty carbon atoms on the periphery of a hollow sphere [13]. Jing and Pan studied the molecular vibrational modes of C60 and C70 through FEM [14]. Application of $\mathrm{C} 60$ in nanocomposites was presented by Khalid et al. via investigation of the properties of C60 buckyballaluminum nanocomposites [15]. They studied the microstructure and interfacial reactions in the mentioned nanocomposite produced by pressurized liquid metal infiltration (squeeze casting) of a tap-packed C60 powder via some experimental techniques.

Carbon nanobuds which can be supposed a newly discovered combination of CNT and C60 is an other important candidate to be used in nanocomposite $[16,17]$. This fact can be attributed to the presence of $\mathrm{C} 60$ molecules on the wall of the CNTs that can act as cleats between CNTs and matrix and prevent from their slips under different loading conditions.

An other important carbon nanostructure is CNC which is the issue of this paper. As mentioned before, the mechanical properties of CNC-based nanocomposites were not studied in detail. But, application of CNCs in

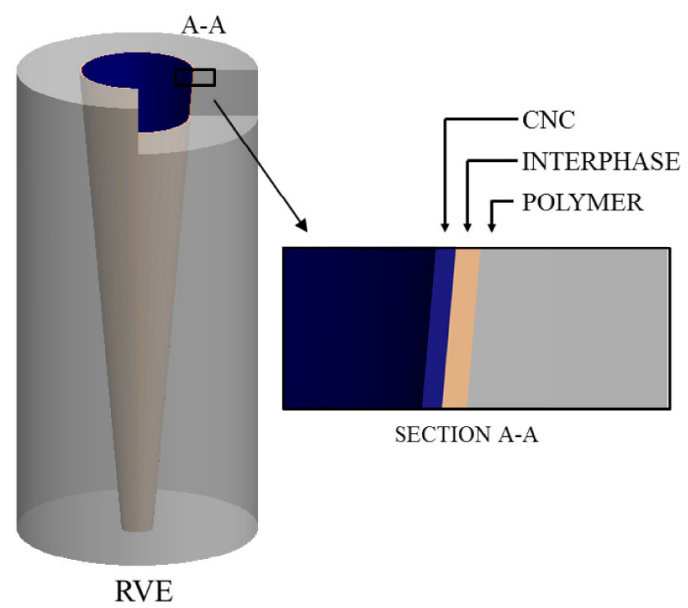

Fig. 1. Schematic representation of the nanocomposite.

nanocomposites can be an interesting field which Momeni and Yassar investigated partially [18]. They studied the stress distribution on a single-walled CNC embedded in an epoxy matrix under a tensile force. Their work was performed using analytical elastic equilibrium equations in cylindrical coordinate system for both of the composite and its inner CNC. They showed that the stress distributions of the CNCs embedded in the matrix were not symmetric and tended to shift toward their tips.

Following the research performed by Momeni and Yasser [18], we are to study the effects of CNCs on the mechanical behaviors of polymer composites more completely. Because their work was only about investigation of stress distribution on CNC-based composite under an axial force, we believe that there are still other main facets related to this nanocomposite which should be studied. The transversely isotropic model under three various loading cases is employed to estimate these effects and evaluate four of five independent constants related to this model. In addition, the variations of these constants vs. different elastic moduli and thicknesses for the polymer matrix are studied in detail. The research is presented for $\mathrm{CNC}$ with $19.2^{\circ}$ apex angle and $0.34 \mathrm{~nm}$ thicknesses. Furthermore, the effects of interphase layer [19] on the mechanical properties of the nanocomposite are studied. Schematic representation of this layer in the interaction surface between the CNC and polymer matrix is shown in Figure 1 and is discussed in the following section.

\section{Formulation and modeling}

Both the CNC and polymer matrix are considered to be in a cylindrical RVE as shown in Figure 2.

There is a layer between the $\mathrm{CNT} / \mathrm{CNC}$ and matrix which is known as interphase layer [20-27]. In order to explain the interaction between the nanotubes and the outer polymer matrix at the atomic level, $\mathrm{Hu}$ et al. conducted the molecular mechanics computations to estimate the thickness of the transition layer in a CNT/polymer composite [22]. Their simulations revealed that after putting 


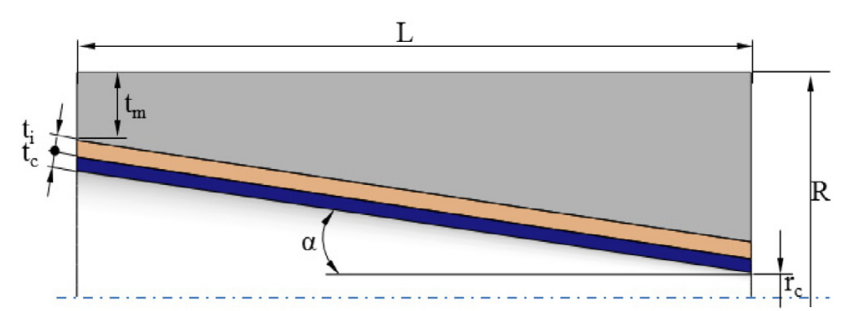

Fig. 2. 2D RVE of a CNC based polymer composite with interphase layer.

Table 1. Elastic constants of the constituent materials of the nanocomposite.

\begin{tabular}{ccc}
\hline Constituent & Elastic modulus (GPa) & Poisson's ratio \\
\hline Polymer matrix & 2.5 & 0.3 \\
CNC & 1000 & 0.3 \\
Interphase layer & 100 & 0.3 \\
\hline
\end{tabular}

the polymer chain closely to the nanotube, the chain started to encircle it. The results proposed that the equilibrium distance between $\mathrm{H}$ atoms of the polymer and $\mathrm{C}$ atoms of the nanotube varies from 0.2851 to $0.5445 \mathrm{~nm}$. Based on this research and [21], we take the average value of this range as the thickness of the interphase layer, i.e., $0.42 \mathrm{~nm}$. Using RVEs to investigate the behaviors of nanocomposites is customary $[5,6,18,19,23,25]$. In fact, the RVE is a simplified model to study the effects of the $\mathrm{CNC}$ and interphase layer on the mechanical behaviors of the polymer matrix. It is assumed that matrix, filer and interphase layer are elastic, isotropic and homogeneous materials which their elastic moduli and Poisson's ratios are given in Table 1.

The length, thickness, half of apex angle and top radius of the CNC are considered $L, t_{c}, \alpha$ and $r_{c}$ respectively whereas the matrix has length $L$ and radius $R$ Moreover, the length and thickness of the interphase layer are supposed $L, t_{i}$ respectively. With the above assumptions, the composite behavior can be modeled as a single transversely isotropic material which has four of five independent constants; namely, two elastic moduli and two Poisson's ratios. The governing equation is the general 3D stress-strain relations in Cartesian or cylindrical coordinate system $[5,19]$ as presented in equation (1). The $z$ axis is supposed to be along with the composite length and two other axes $(x, y)$ are considered on the circular cross section.

$$
\left\{\begin{array}{l}
\varepsilon_{x} \\
\varepsilon_{y} \\
\varepsilon_{z}
\end{array}\right\}=\left[\begin{array}{ccc}
\frac{1}{E_{x}} & -\frac{v_{x y}}{E_{x}} & -\frac{v_{z x}}{E_{z}} \\
-\frac{v_{x y}}{E_{x}} & \frac{1}{E_{x}} & -\frac{v_{z x}}{E_{z}} \\
-\frac{v_{z x}}{E_{z}} & -\frac{v_{z x}}{E_{z}} & \frac{1}{E_{z}}
\end{array}\right]\left\{\begin{array}{l}
\sigma_{x} \\
\sigma_{y} \\
\sigma_{z}
\end{array}\right\}
$$

where, $\varepsilon_{x}=\frac{\Delta x}{R}=\varepsilon_{y}=\frac{\Delta y}{R}$ and $\varepsilon_{z}=\frac{\Delta z}{L}$ are respectively strains along $x, y$ and $z$ directions, $R$ is the radius of the RVE, $\sigma_{x}, \sigma_{y}$ and $\sigma_{z}$ are stresses along $x, y$ and $z$ coordinates and finally $E_{x}, E_{z}, v_{x y}$ and $v_{z x}$ are elastic moduli and Poisson's ratios along coordinates determined by

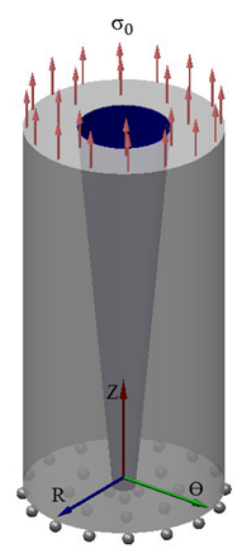

(a)

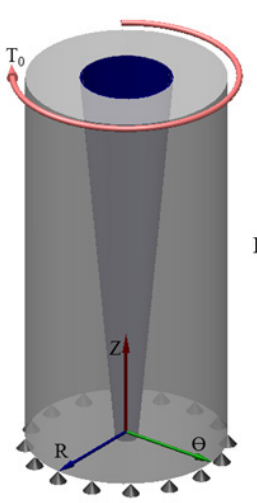

(b)

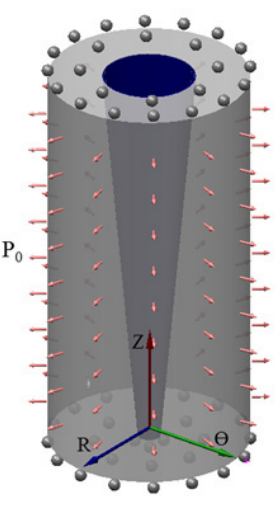

(c)
Fig. 3. Three required loading conditions to obtain effective constants of the composite.

their subscripts. This equation can be generalized for the cylindrical coordinate system which $z$ axis is along with the RVE axis and the two other directions, radial and tangential coordinates $(r, \theta)$ are on the cross section as depicted in Figure 3.

For cylindrical coordinate system, $\varepsilon_{\theta}=\frac{\Delta R}{R}, v_{x y}=v_{r \theta}$ and $v_{z x}=v_{z r}=v_{z \theta}$, where $v_{r \theta}$, and $v_{z r}$ are respectively the Poisson's ratios along their subscripts. Finally, $E_{r}$ in cylindrical coordinate system is the elastic modulus along radial direction. In order to estimate four of five constants, the RVE should be under at least three different loading conditions. The procedure of evaluating the coefficients under various loading conditions is to be discussed below $[5,6]$.

\subsection{Cylindrical RVE under an axial loading}

In this loading case, as illustrated in Figure 3a, the applied stress is axial and does not have any component along $x$ and $y$ (or $r$ and $\theta$ ) directions. Therefore, the stress and strain components at any point on the surface of the RVE are:

$$
\begin{aligned}
& \sigma_{x}=\sigma_{y}=\sigma_{r}=\sigma_{\theta}=0 \\
& \varepsilon_{z}=\frac{\Delta L}{L}, \quad \varepsilon_{\theta}=\frac{\Delta R}{R}
\end{aligned}
$$

where, $\Delta R$ is the radial (lateral) displacement under an axial force. From the third relation in equation (1), we have:

$$
E_{z}=\frac{\sigma_{z}\left(=\sigma_{0}\right)}{\varepsilon_{z}}=\frac{L}{\Delta L} \sigma_{\text {ave }}
$$

where, $\sigma_{\text {ave }}$ is the averaged stress along $z$ direction that can be estimated for the RVE through FEM.

Furthermore, from equation (1) (in cylindrical coordinate system) and above relations, we can write:

$$
\varepsilon_{\theta}=-\frac{v_{z x}}{E_{z}} \sigma_{z}=-v_{z x} \frac{\Delta L}{L}=\frac{\Delta R}{R}
$$


Therefore, from above equation, we obtain:

$$
v_{z x}=v_{z r}=-\left(\frac{\Delta R}{R}\right) /\left(\frac{\Delta L}{L}\right)
$$

Using FEM, we can compute $\Delta R$ and then equations (2) and (3) can be used to estimate the elastic modulus $E_{z}$ and Poisson's ratio $v_{z x}\left(=v_{z r}\right)$. Hence, with the first loading case, we can estimate the two constants out of the four of five effective constants of the transversely isotropic RVE. Other constants are evaluated from other loading cases which will be studied in the following subsections.

\subsection{Cylindrical RVE under a torsional torque}

This loading case is shown in Figure 3b. As shown in this figure, the axisymmetric RVE is under an antisymmetric torsional torque. The torsional angle of the free end of the RVE can be calculated via equation (4).

$$
\begin{gathered}
\varphi=\frac{T_{0} L}{G_{x y} J}, \text { with } \quad G_{x y}=\frac{E_{x}}{2\left(1+v_{x y}\right)} \\
\Rightarrow \frac{E_{x}}{2\left(1+v_{x y}\right)}=G_{x y}\left(=\frac{T_{0} L}{J \varphi}\right)
\end{gathered}
$$

where,

$$
\varphi, T_{0}, G_{x y}\left(=G_{r \theta}\right), J, E_{x}\left(=E_{r}=E_{\theta}\right)
$$

and

$$
v_{x y}\left(=v_{r \theta}\right)
$$

are respectively torsional angle, applied torsional torque, shear modulus, moment of inertia of the cross section, elastic modulus along radial (lateral) direction and Poisson's ratio in $(x, y)$ or $(r, \theta)$ plane.

Estimating $\varphi$ resulted from the applied $T_{0}$ from FEM, there remain two unknown constants in equation (4):

$$
E_{x}\left(=E_{r}=E_{\theta}\right) \quad \text { and } \quad v_{x y}\left(=v_{r \theta}\right)
$$

\subsection{Cylindrical RVE under a uniform radial pressure}

In this case, the RVE is constrained in axial direction from both ends and under a radial (lateral) uniform pressure as shown in Figure 3c. Due to the type of this loading case the strain in $z$ direction $\varepsilon_{z}$ is zero and then the plane strain condition is maintained. Displacement along the radial direction is $\frac{\Delta R}{R}$ which can be estimated from FEM. From the third relation of equation (1), we have: $\sigma_{z}=v_{z x}\left(\sigma_{\theta}+\sigma_{r}\right)$. Thus, on the outer surface of the RVE which $\sigma_{r}=P_{0}$, the following formula can be derived from equation (1):

$$
\varepsilon_{\theta}=-\left(\frac{v_{x y}}{E_{x}}+\frac{v_{z x}^{2}}{E_{z}}\right) \sigma_{r}+\left(\frac{1}{E_{x}}-\frac{v_{z x}^{2}}{E_{z}}\right) \sigma_{\theta}=\frac{\Delta R}{R}
$$

If $\sigma_{\theta}$ and $\Delta R$ are evaluated from FEM there are only two unknown constants $E_{x}\left(=E_{r}=E_{\theta}\right)$ and $v_{x y}\left(=v_{r \theta}\right)$ With attention to the unknown values in the previous loading case (torsional torque) and this loading case (radial pressure), we find that the unknown constants are same and then, they can be calculated from concurrently solving of equations (4) and (5).

\section{Results and discussion}

The above modeling approaches were performed on different RVEs in the presence of interphase layer including different polymers as matrices and several values for thicknesses of a certain polymer. The results are to be presented in this section. The considered numerical values of the elastic moduli and Poisson's ratios of the CNC, polymer matrix and interphase layer are reported in Table $1[6,19,20,27]$. The motivation to estimate the elastic modulus of the interphase layer equaling $100 \mathrm{GPa}$ is that this value is not actually a fixed value. As discussed in reference [21], it depends on the material of the polymer matrix. For example, for the CNT/PMMA composite the value was reported $93.67 \mathrm{GPa}[21]$. For other polymers, it may be a bit larger or smaller. Since we do not want to limit our research for a certain polymer, it was considered $100 \mathrm{GPa}$ (compared to 93.67 GPa). In addition, the CNC, in this paper, is considered as an isotropic material with the same elastic properties in all directions. However, the $\mathrm{CNT}$ and $\mathrm{CNC}$ are not actually isotropic materials and this is a simplifying assumption [5-7, 19,21,26,27]. Moreover, the thickness of the $\mathrm{CNC}$ is considered $0.34 \mathrm{~nm}$ and the smaller thickness of the matrix $\left(t_{m}\right.$ in Fig. 1) varies from $3 \mathrm{~nm}$ to $10 \mathrm{~nm}$. These values are fed into FEM to compute the four of five unknown constants. The FEM is extensively used in nano modeling and for this special case, nanocomposite material, other researchers, for example, references [5-7, 19,21,26,27], applied this method. Applying described three loading cases, we can estimate the constants of the nanocomposite based on the explanations discussed earlier. The results are presented for various matrix elastic moduli and thicknesses in the presence of interphase layer.

\subsection{Determination of constants for different matrix elastic moduli}

In this section, the discussed four of five constants are evaluated for different matrix elastic moduli. In this modeling, the thickness of the polymer matrix is supposed $3 \mathrm{~nm}$ and its elastic modulus varies from 2.5-100 GPa Also, the apex angle and thickness of the CNC are considered $19.2^{\circ}$ and $0.34 \mathrm{~nm}$. As mentioned before, the thickness of the interphase layer is $0.42 \mathrm{~nm}$. The variations of $E_{z} / E_{m}$ and $E_{r} / E_{m}$ vs. matrix elastic moduli are depicted in Figure 4.

The subscript " $i$ " denotes the behaviors of the nanocomposite in the presence of interphase layer. This figure shows that the presence of this layer increases both of the mentioned ratios. Of course, we do not change the values of the elastic modulus of the interphase layer with increasing the elastic modulus of the matrix. With increasing the elastic modulus of the interphase layer, we will certainly observe higher values for the discussed ratios. Furthermore, the figure proposes that all of the mentioned parameters decrease remarkably with increasing the elastic modulus of the matrix. Of course, these are 


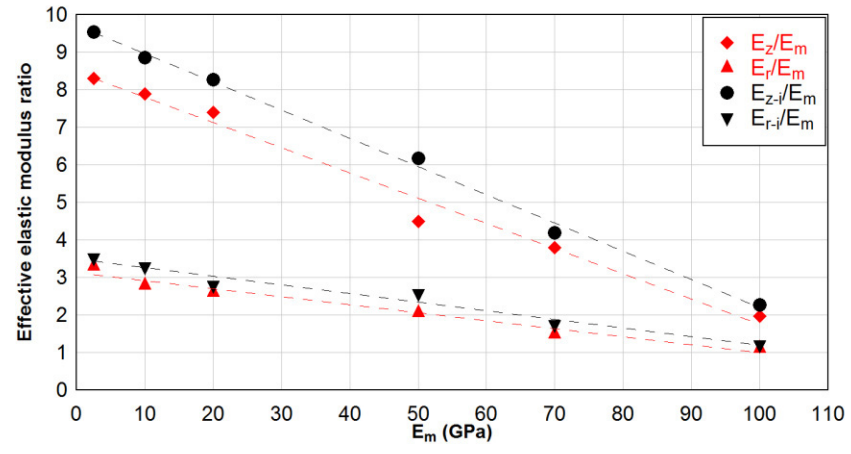

Fig. 4. Variations of $E_{z} / E_{m}$ and $E_{r} / E_{m}$ vs. matrix elastic moduli.

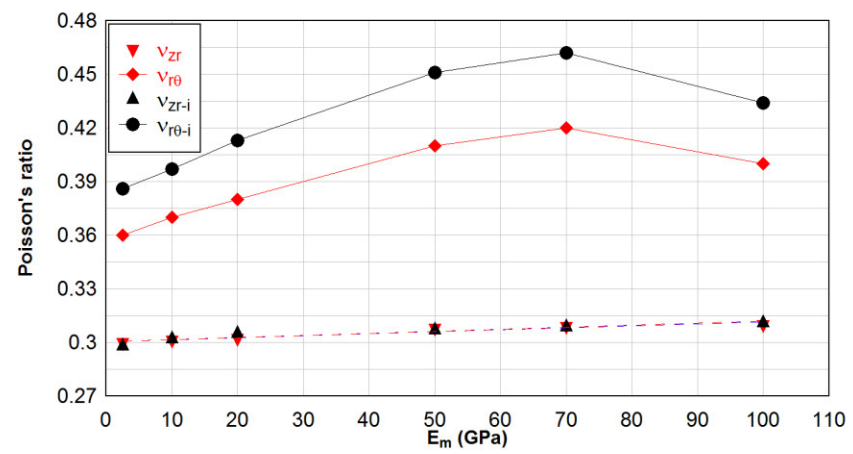

Fig. 5. Variations of the Poisson's ratios of the RVE vs. the elastic modulus of the matrix.

ratios and it is worth noting that the values of axial and radial elastic moduli of the RVE increase with increasing the elastic modulus of each constituent if matrix or filler. The main point is that with increasing the elastic modulus of the polymer, the mentioned ratios approach each other. Therefore, in this loading case the gradient of variations decreases with increasing the matrix elastic modulus and the axial and radial elastic moduli of the RVE become closer to each other.

On the other hand, Figure 5 depicts the variations of the Poisson's ratios in two $z r$ or $r \theta$ planes vs. the elastic modulus of the matrix.

In this case, the figure proposes that the presence of the interphase layer does not have any effective influence on $v_{z r}$ whereas it increases $v_{z \theta}$. In addition, increasing the elastic modulus of the matrix does not affect $v_{z r}$ tremendously. On the contrary, $v_{r \theta}$ increases with increasing the elastic modulus of the matrix up to a maximum value and decreases after it. The effect of the interphase layer on $v_{z r}$ increases with increasing the elastic modulus of the matrix up to a certain value that is shown in Figure 5. For this case, according to the figure, $E_{m}=70 \mathrm{GPa}$ and $v_{r \theta}=0.42$ is the maximum point for without interphase layer and $E_{m}=70 \mathrm{GPa}$ and $v_{r \theta}=0.42$ is maximum point for the model with this layer that is closer to the real value.

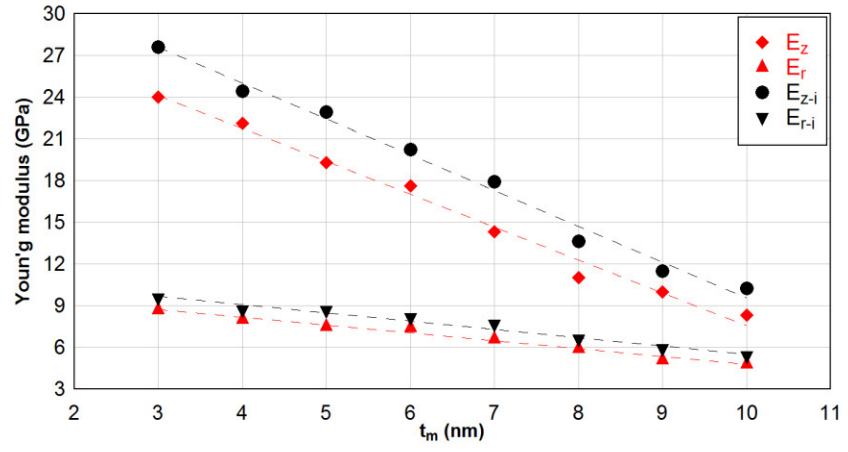

Fig. 6. Variations of the axial and radial elastic moduli of the RVE vs. matrix thickness.

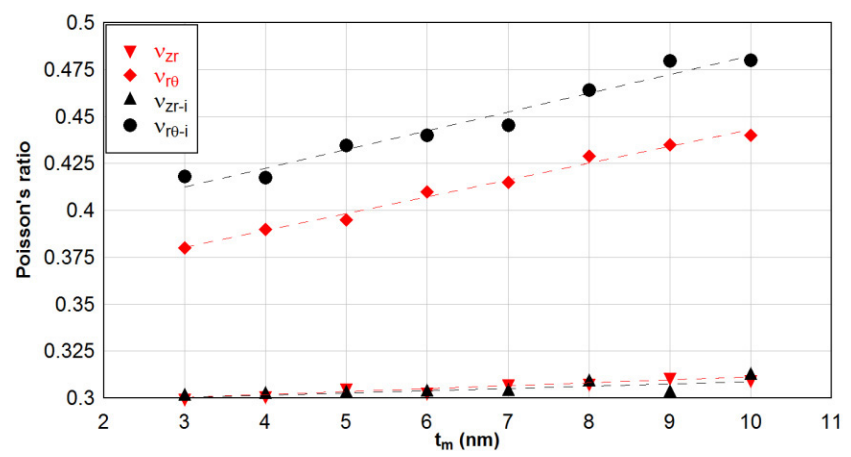

Fig. 7. Variations of the Poisson's ratios of the RVE vs. the matrix thickness.

\subsection{Determination of constants for different matrix thicknesses}

In this subsection, the variations of the four of five constants of the RVE vs. the thickness of the matrix are investigated. The values of CNC angle and interphase layer elastic modulus are the same as the previous case and the elastic modulus of the matrix is considered 2.5 GPa.

Figure 6 illustrates the values of axial and radial elastic moduli of the RVE vs. variations of the thickness of the polymer matrix.

The figure shows that the existence of the interphase layer does not have remarkable effects on radial elastic modulus of the RVE in comparison with the axial elastic modulus. In addition, it proposes that with increasing the thickness of the elastic matrix, the values of both of the elastic moduli decrease Of course, the variation in the RVE axial elastic modulus is more appreciable. The important point is that with increasing the thickness of the matrix, the values of the elastic moduli approach each other.

On the other hand, Figure 7 demonstrates the variations of the Poisson's ratios in $z r$ and $r \theta$ planes vs. the thickness of the matrix.

The results show that the presence of the interphase layer does not affect $v_{z r}$ at all whereas it increases $v_{r \theta}$. Moreover, $v_{r \theta}$ increases with increasing the thickness of the matrix greatly. In contrast, the variations of the matrix thickness does not have effective influence on the values of $v_{z r}$. Thus, it can be concluded that the value 
of $v_{z r}$ is approximately independent of the matrix thickness value.

\section{Conclusion}

In this paper, the elastic behaviors of a transversely isotropic polymer composite with CNC fillers were studied. The RVEs including matrix, CNC ad interphase layer extracted from the nanocomposite were situated under some loading cases to evaluate their four of five constants related to the motioned model. In addition, the effects of different elastic moduli and thicknesses for matrix on the four of five elastic constants were investigated. In general, the results proposed that the presence of interphase layer which is closer to the real conditions increases the elastic properties of the CNC-base polymer nanocomposites and it can be considered as a main distinction between carbon nanostructures as fillers with others.

\section{References}

[1] F.H. Su, Z.Z. Zhang, K. Wang, W. Jiang, X.H. Men, W.M. Liu, Friction and wear properties of carbon fabric composites filled with nano- $\mathrm{Al}_{2} \mathrm{O}_{3}$ and nano-Si $\mathrm{N}_{4}, \mathrm{~J}$. Compos. Part A: Appl. Sci. Manufact. 37 (2006) 13511357

[2] X. Huang, A. Netravali, Characterization of flax fiber reinforced soy protein resin based green composites modified with nano-clay particles, J. Compos. Sci. Tech. 67 (2007) 2005-2014

[3] F.H. Su, Z.Z. Zhang, W.M. Liu, Tribological and mechanical properties of Nomex fabric composites filled with polyfluo 150 wax and nano- $\mathrm{SiO}_{2}$, Compos. Sci. Tech. 67 (2007) 102-110

[4] M.M.R. Taha, A.B. Colak-Altunc, M. Al-Haik, A multiobjective optimization approach for design of blastresistant composite laminates using carbon nanotubes, J. Compos. B 40 (2009) 522-529

[5] Y.J. Liu, X.L. Chen, Evaluations of the effective material properties of carbon nanotube-based composites using a nanoscale representative volume element, J. Mech. Mater. 35 (2003) 69-81

[6] X.L. Chen, Y.J. Liu, Square representative volume elements for evaluating the effective material properties of carbon nanotube-based composites, J. Comput. Mater. Sci. 29 (2004) 1-11

[7] M.M. Shokrieh, R. Rafiee, Investigation of nanotube length effect on the reinforcement efficiency in carbon nanotube based composites, J. Compos. Struct. 92 (2010) $2415-2420$

[8] V.V. Mokashi, D. Qian, Y. Liu, A study on the tensile response and fracture in carbon nanotube-based composites using molecular mechanics, J. Compos. Sci. Tech. 67 (2007) 530-540

[9] G. Formica, W. Lacarbonara, R. Alessi, Vibrations of carbon nanotube-reinforced composites J. Sound Vib. 329 (2010) 1875-1889

[10] L.L. Ke, J. Yang, S. Kitipornchai, Nonlinear free vibration of functionally graded carbon nanotube-reinforced composite beams, J. Compos. Struct. 92 (2010) 676-683
[11] K. Fukuchi, K. Sasaki, K. Katagiri, T. Imanishi, A. Kakitsuji, Aluminium based high thermal conductive composite containing CNT and VGCF-deformation dependence of thermal conductivity, Procedia Eng. 10 (2011) 912-917

[12] P.E. Lopes, F.V. Hattum, C.M.C. Pereira, P.J.R.O. Novoa, S. Forero, F. Hepp, L. Pambaguian, High CNT content composites with CNT Buckypaper and epoxy resin matrix: Impregnation behavior composite production and characterization, Compos. Struct. 92 (2010) $1291-1298$

[13] W. Krätschmer, L.D. Lamb, K. Fostiropoulos, D.R. Huffman, C60: a new form of carbon, Nature 347 (1990) 354-358

[14] Du Jing, Zeng Pan, Molecular vibrational modes of C60 and C70 via finite element method, Eur. J. Mech. A/ Solids. 28 (2009) 948-954

[15] F.A. Khalid, O. Beffort, U.E. Klotz, B.A. Keller, P. Gasser, S. Vaucher, J. Acta Mat. 51 (2003) 4575

[16] 14. A.G. Nasibulin, P.V. Pikhitsa, H. Jiang, D.P. Brown, A.V. Krashninnikov, A.S. Anisimov, P. Queipo, A. Moisala, D. Gonzalez, G.N. Lientschnig, A. Hassanein, S.D. Shandakov, G. Lolli, D.E. Resasco, M. Chio, D.T. Nek, E.I. Kauppinen, A novel hybrid carbon material, Nat. Nanotech. 2 (2007) 156-161

[17] X. Wu, X.C. Zeng, First-Principles Study of a Carbon Nanobud, ACS Nano. 2 (2008) 1459-1465

[18] K. Momeni, R.S. Yassar, Stress distribution on a singlewalled carbon nanohorn embedded in an epoxy matrix nanocomposite under axial force, J. Comput. Theor. Nanosci. 7 (2010) 1-7

[19] H. Golestanian, M. Shojaie, Numerical characterization of CNT-based polymer composites considering interface effects, Comput. Mater. Sci. 50 (2010) 731-736

[20] A. Hernandez-Perez, F. Aviles, Modeling the influence of interphase on the elastic properties of carbon nanotube composites, Comput. Mater. Sci. 47 (2010) 926-933

[21] A. Montazeri, 1 R. Naghdabadi, Investigation of the interphase effects on the mechanical behavior of carbon nanotube polymer composites by multiscale modeling, J. Appl. Polymer Sci. 117 (2010) 361-367

[22] N. Hu, H. Fukunaga, C. Lu, M. Kameyama, B. Yan, Prediction of elastic properties of carbon nanotubereinforced composites, Proc. R. Soc. 461 (2005) 16851710

[23] P. Joshi, S.H. Upadhyay, Effect of interphase on elastic behavior of multiwalled carbon nanotube reinforced composite, Comput. Mater. Sci. 87 (2014) 267-273

[24] P. Lu, Y.W. Leong, P.K. Pallathadka, C.B. He, Effective moduli of nanoparticle reinforced composites considering interphase effect by extended double-inclusion modelTheory and explicit expressions, Int. J. Eng. Sci. 73 (2013) 33-55

[25] K.N. Spanos, S.K. Georgantzinos, N.K. Anifantis, Investigation of stress transfer in carbon nanotube reinforced composites using a multi-scale finite element approach, Compos. Part B: Eng. 63 (2014) 85-93

[26] R. Rafiee, R. Pourazizi, Influence of CNT functionalization on the interphase region between CNT and polymer, Comput. Mater. Sci. (2014) in press

[27] P. Kumar, J. Srinivas, Numerical evaluation of effective elastic properties of CNT-reinforced polymers for interphase effects, Comput. Mater. Sci. 88 (2014) 139-144 